\title{
Erratum to: Resuscitative endovascular balloon occlusion of the aorta versus aortic cross clamping among patients with critical trauma: a nationwide cohort study in Japan
}

Toshikazu Abe ${ }^{1,2^{*}}$, Masatoshi Uchida', Isao Nagata' ${ }^{1}$, Daizoh Saitoh ${ }^{3}$ and Nanako Tamiya ${ }^{1}$

\section{Erratum}

Following publication of the original article [1], it was brought to our attention that there were a few errors in Table 2:

CU admission should read: ICU admission

$11 / 233$ (1.8\%) should read: 11/233 (4.7\%)

12/233 (2.0\%) should read: $12 / 233$ (5.2\%)

The corrected table is presented in this erratum [Table 2].

Furthermore, the sentence "...only 14\% (83/607) of REBOA patients and $2.0 \%(12 / 233)$ of ACC patients could leave the hospital and go home." in the Discussion section should as a consequence read: "...only $14 \%(83 / 607)$ of REBOA patients and 5.2\% (12/233) of ACC patients could leave the hospital and go home."

This has now been corrected in this erratum.

\footnotetext{
Author details

'Department of Health Services Research, Faculty of Medicine, University of Tsukuba, 1-1-1, Tennodai, Tsukuba 305-8577, Japan. ${ }^{2}$ Department of Emergency Medicine, Tsukuba Medical Center Hospital, 1-3-1, Amakubo, Tsukuba 305-8558, Japan. ${ }^{3}$ Department of Traumatology and Emergency Medicine, National Defense Medical College, 3-2, Namiki, Tokorozawa, Saitama 359-8513, Japan.
}

Received: 8 February 2017 Accepted: 10 February 2017

Published online: 22 February 2017

\section{Reference}

1. Abe T, Uchida M, Nagata I, Saitoh D, Tamiya N. Resuscitative endovascular balloon occlusion of the aorta versus aortic cross clamping among patients with critical trauma: a nationwide cohort study in Japan. Crit Care. 2015;20: 400. doi:10.1186/s13054-016-1577-x.

\footnotetext{
* Correspondence: abetoshi111@gmail.com

'Department of Health Services Research, Faculty of Medicine, University of Tsukuba, 1-1-1, Tennodai, Tsukuba 305-8577, Japan

${ }^{2}$ Department of Emergency Medicine, Tsukuba Medical Center Hospital,

1-3-1, Amakubo, Tsukuba 305-8558, Japan

Full list of author information is available at the end of the article
} 
Table 2 Outcome comparisons between REBOA and ACC

\begin{tabular}{llll}
\hline & REBOA $(n=636)$ & ACC $(n=267)$ & $P$ value \\
\hline Disposition at discharge & & & $<0.001^{*}$ \\
Died (in-hospital mortality) & $405 / 607(67 \%)$ & $210 / 233(90 \%)$ & \\
Transferred & $118 / 607(19 \%)$ & $11 / 233(4.7 \%)$ & \\
Home & $83 / 607(14 \%)$ & $12 / 233(5.2 \%)$ & \\
Other & $1 / 607(0.1 \%)$ & $0 / 233(0.0 \%)$ & \\
Disposition at ED & & & $<0.001^{*}$ \\
Died (ED mortality) & $137 / 625(22 \%)$ & $130 / 264(49 \%)$ & \\
ICU admission & $472 / 625(76 \%)$ & $129 / 264(49 \%)$ & \\
Ward admission & $137 / 625(22 \%)$ & $4 / 264(1.5 \%)$ & \\
Other & $5 / 625(1.8 \%)$ & $1 / 264(0.4 \%)$ & \\
\hline
\end{tabular}

The variables are shown as $n(\%)$

$A C C$ aortic cross clamping, ED emergency department, ICU intensive care unit, $R E B O A$ resuscitative endovascular balloon occlusion of the aorta

"Chi-square test 